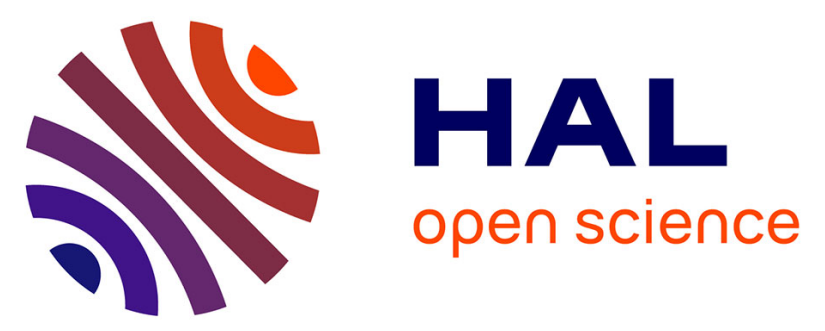

\title{
High diversity of non-sporulating moulds in respiratory specimens of immunocompromised patients: should all the species be reported when diagnosing invasive aspergillosis?
}

Dea Garcia-Hermoso, Alexandre Alanio, Odile Cabaret, Martine Olivi, Françoise Foulet, Catherine Cordonnier, Jean-Marc Costa, Stéphane Bretagne

\section{To cite this version:}

Dea Garcia-Hermoso, Alexandre Alanio, Odile Cabaret, Martine Olivi, Françoise Foulet, et al.. High diversity of non-sporulating moulds in respiratory specimens of immunocompromised patients: should all the species be reported when diagnosing invasive aspergillosis?. Mycoses, 2015, 58 (9), pp.557-564. $10.1111 /$ myc. 12356 . pasteur-01405227

\section{HAL Id: pasteur-01405227}

\section{https://hal-pasteur.archives-ouvertes.fr/pasteur-01405227}

Submitted on 29 Nov 2016

HAL is a multi-disciplinary open access archive for the deposit and dissemination of scientific research documents, whether they are published or not. The documents may come from teaching and research institutions in France or abroad, or from public or private research centers.
L'archive ouverte pluridisciplinaire HAL, est destinée au dépôt et à la diffusion de documents scientifiques de niveau recherche, publiés ou non, émanant des établissements d'enseignement et de recherche français ou étrangers, des laboratoires publics ou privés.

\section{(ㄷ)(1) $\$$}

Distributed under a Creative Commons Attribution - NonCommerciall 4.0 International 


\title{
High diversity of non-sporulating moulds in respiratory specimens of immunocompromised patients: should all the species be reported when diagnosing invasive aspergillosis?
}

\author{
Dea Garcia-Hermoso, ${ }^{1,2}$ Alexandre Alanio, ${ }^{1,2,3,4}$ Odile Cabaret, $^{5,6}$ Martine Olivi, $^{7}$ Françoise Foulet, ${ }^{5}$ \\ Catherine Cordonnier, ${ }^{6,8}$ Jean-Marc Costa ${ }^{7}$ and Stéphane Bretagne ${ }^{1,2,3,4}$ \\ ${ }^{1}$ Institut Pasteur, Molecular Mycology Unit, National Reference Center of Invasive Mycoses and Antifungals, Paris, France, ${ }^{2}$ CNRS URA3012, Paris, France, \\ ${ }^{3}$ Laboratoire de Parasitologie-Mycologie, Groupe hospitalier Lariboisière-Saint Louis, Assistance Publique-Hôpitaux de Paris (APHP), Paris, France, ${ }^{4}$ Sorbonne \\ Paris Cité, Université Paris Diderot, Paris, France, ${ }^{5}$ Laboratoire de Parasitologie-Mycologie, Groupe hospitalier Albert Chenevier-Henri Mondor, Assistance \\ Publique-Hôpitaux de Paris (APHP), Créteil, France, ${ }^{6}$ Université Paris-Est-Créteil, Créteil, France, 7 Laboratoire CERBA, Cergy Pontoise, France and \\ ${ }^{8}$ Département d'Hématologie, Groupe Hospitalier Albert Chenevier-Henri Mondor, Assistance Publique-Hôpitaux de Paris (APHP), Paris, France
}

\section{Summary}

\begin{abstract}
Non-sporulating moulds (NSMs) isolated from respiratory specimens are usually discarded without further testing although they may have pathogenic effects in immunocompromised patients. The objective of this study was to determine the identity and frequency of NSMs in patients with haematological malignancies. We analysed the mycological results of 251 consecutive respiratory samples from 104 haematology patients. Yeast and sporulating moulds were identified at the genus/ species level according to their phenotypic features. NSMs were identified by internal transcribed spacer (ITS) sequencing. We detected 179 positive samples, of which $10.1 \%$ (18/179) were mixtures of moulds and $26.3 \%$ (47/179) were mixtures of moulds and yeast. We identified 142 moulds belonging to 11 different genera/species or groups, with Aspergillus fumigatus $(n=50)$, Penicillium spp. $(n=31)$ and NSM $(n=24)$ being the most frequently isolated species. Twenty-two NSMs were successfully sequenced: 18 were basidiomycetes and six were ascomycetes, corresponding to 16 different genera/species. NSMs were isolated with A. fumigatus in the same sample or in a subsequent sample in five patients with probable invasive aspergillosis. The conclusion is that the respiratory specimens of immunocompromised patients frequently contain very diverse mould species that may increase the virulence of pathogenic species. Reporting all mould species isolated when diagnosing invasive fungal infection could test this hypothesis.
\end{abstract}

Key words: Respiratory specimens, non-sporulating mould, immunocompromised patient, invasive aspergillosis.

\section{Introduction}

Invasive mould diseases (IMD) are the main cause of fungal disease in immunocompromised patients with

Correspondence: Stéphane Bretagne, Laboratoire de ParasitologieMycologie, Hôpital Saint Louis, 1 rue Claude Vellefaux, 75010, Paris, France. Tel.: +331424995 03. Fax: +33142494803.

E-mail: stephane.bretagne@sls.aphp.fr

Submitted for publication 27 March 2015

Revised 20 June 2015

Accepted for publication 21 June 2015 haematological disorders. In epidemiological and therapeutic studies, IMD are diagnosed according to consensus criteria defined by the European Organization for Research and Treatment of Cancer/Mycosis Study Group (EORTC/MSG). ${ }^{1}$ These criteria include the recovery of a mould in cultures of non-sterile specimens such as sputum, broncho-alveolar lavage fluid, bronchial brush or sinus aspirates. Such samples are easily obtained and can lead to the diagnosis of probable IMD. ${ }^{1}$ The EORTC/MSG criteria do not grade the identified mould according to whether it was isolated as a pure colony and/or from repeat samples. 
Furthermore, the mould species included in the EORTC/MSG criteria are not clearly defined, although it is usually accepted that only species with known pathogenic potential should be considered when analysing respiratory specimens. This includes Aspergillus, Fusarium, Mucorales and Scedosporium spp., with Aspergillus fumigatus being by far the main species responsible for invasive aspergillosis (IA). ${ }^{2-4}$ Apart from these well-+known pathogenic moulds, other moulds isolated from respiratory specimens are usually deemed at best colonising moulds or irrelevant environmental contaminants. This prevents all further analysis and even the reporting of such moulds.

To challenge this attitude, we analysed the results of mycological cultures of respiratory specimens obtained from haematology patients during routine practice in our laboratory. We identified non-sporulating moulds (NSMs) by sequencing the nuclear ribosomal internal transcribed spacer (ITS) region as consensually proposed. ${ }^{5}$ Sequencing can identify putative emergent pathogenic species without a priori. ${ }^{6}$ On the other hand, pathogenic moulds without reproductive structures, such as non-sporulating A. fumigatus, ${ }^{7}$ may be falsely discarded as contaminants. The goal of this study was to describe the diversity of fungi recovered from cultured respiratory specimens from haematology patients, focusing on non-sporulating moulds. In particular, we sought to determine how often such moulds are isolated pure or in association with other fungi.

\section{Materials and methods}

\section{Respiratory specimens}

We performed a prospective single-centre hospitalbased study including every sputum and broncho-tracheal aspirate received in our laboratory from June 15, 2010 to December 31, 2011 as part of routine laboratory testing for suspected IMD in the context of pneumonia and/or unexplained fever in patients with haematological malignancies. Most broncho-tracheal aspirates were obtained by bronchoalveolar lavage. Patients were definitively classified into probable IMD or invasive aspergillosis (IA) according to the 2008 EORTC/MSG criteria ${ }^{1}$ every 2 months during a multidisciplinary medical meeting. The clinicians were aware of the presence of NSM, but not of the molecular analysis; therefore, our findings did not influence any medical decisions. According to French Public Health Law (CSP Art L1121-1.1), our study protocol required neither the approval of an ethics committee nor informed consent for the use of patient samples.
According to standard practice in our laboratory, following their arrival, respiratory samples were centrifuged and seeded onto (i) one plate containing chromogenic medium (Candiselect 2 BioMérieux, Marcy l'Etoile, France) incubated at $37{ }^{\circ} \mathrm{C}$ for 1 week, and (ii) two Sabouraud agar slants supplemented with chloramphenicol and gentamicin (Bio-Rad, Marnes la Coquette, France) incubated at $37{ }^{\circ} \mathrm{C}$ and at $30{ }^{\circ} \mathrm{C}$ (one at each temperature) for 3 weeks. Cultures were checked daily and each colony was identified using ID32C (BioMérieux) for yeast, and macro and microscopic phenotypic features for moulds according to standard practices. ${ }^{8}$ If several colonies were observed, each colony was seeded onto a new plate to obtain pure colonies. In the absence of fructification suggestive of a species or a genus, the colony was categorised as NSM.

\section{DNA extraction, amplification and sequencing}

The NSM colony was scraped from the agar slant, transferred to $1 \mathrm{ml}$ of PBS, vortexed for $60 \mathrm{~s}$ and then heated for $15 \mathrm{~min}$ at $100{ }^{\circ} \mathrm{C}$. The suspension was vortexed briefly and then pelleted for $5 \mathrm{~min}$ at a maximum speed of $16000 \mathrm{~g}$ in a microcentrifuge. DNA was extracted from the supernatant using the Qiagen DNA Blood kit (QIAgen, Courtaboeuf, France) according to the manufacturer's recommendations. DNA was finally eluted in $50 \mu \mathrm{l}$ of the elution buffer and quantified using a NanoDrop DN-1000 Spectrophotometer (Fisher Scientific, Illkirch, France).

A total of $10 \mathrm{ng}$ of DNA was amplified for both the ITS1 and ITS2 regions in two independent reactions in a final volume of $50 \mu \mathrm{l}$. An M13 tail was added to the ITS primers $^{9}$ to standardise the amplification and sequencing parameters (ITS5-M13F: 5'-GTAGCGCAG CGGCCAGTGGAAGTAAAAGTCGTAACAAGG-3'; ITS2M13R: 5'-CAGGGCGCAGCGATGACGCTGCGTTCTTCA TCGATGC-3'; ITS3-M13F: 5'-GTAGCGCAGCGGCCAGT GCATCGATGAAGAACGCAGC-3'; ITS4-M13R: 5-CA GGGCGCAGCGATGACTCCTCCGCTTATTGATATGC-3'). The amplification reaction mixture consisted of $1 \mathrm{X}$ Fast Start PCR Buffer, $3 \mathrm{mmol} \mathrm{L}^{-1} \quad \mathrm{MgCl}_{2}$, $0.2 \mathrm{mmol} \mathrm{L}^{-1}$ of each dNTP, $0.2 \mu \mathrm{mol} \mathrm{L}{ }^{-1}$ of each primer and $1.25 \mathrm{U}$ of Fast Start Taq DNA polymerase (Roche Diagnostics, Meylan, France). After an initial denaturation step at $95{ }^{\circ} \mathrm{C}$ for $8 \mathrm{~min}$, samples were amplified for 35 cycles of denaturation at $95{ }^{\circ} \mathrm{C}$ for $30 \mathrm{~s}$, annealing at $52{ }^{\circ} \mathrm{C}$ for $30 \mathrm{~s}$ and extension at 72 ${ }^{\circ} \mathrm{C}$ for $1 \mathrm{~min}$, followed by a final extension at $72{ }^{\circ} \mathrm{C}$ for $5 \mathrm{~min}$. PCR products were visualised on a QIAxcel 
instrument (QIAgen) and then enzymatically cleaned with shrimp alkaline phosphatase (SAP) and exonuclease I (Exo) to remove un-incorporated dNTPs and primers, respectively. A total of $3 \mu \mathrm{l}$ of corresponding PCR reaction was incubated with $2 \mu \mathrm{l}$ of ExoSAP-IT (USB Europe, Staufen, Germany) for $30 \mathrm{~min}$ at $37^{\circ} \mathrm{C}$ followed by $15 \mathrm{~min}$ at $80^{\circ} \mathrm{C}$ to inactivate the enzyme, and was then sequenced bidirectionally using the Dideoxy Terminator Cycle Sequencing Kit v1.1 protocol (Applied Biosystems, Courtaboeuf, France). The reaction products were purified by gel filtration using Sephadex G-50 (Amersham, Courtaboeuf, France), run on an ABI PRISM 3130 Genetic Analyser and analysed with the Seoscape v2.6 software (Applied Biosystems, Courtaboeuf, France).

The obtained sequences were subjected to pairwise alignments against curated fungal reference databases available at the on-line MycoBank database (http:// www.mycobank.org/). In addition, a BLAST search against known sequences in NCBI Genbank was performed. Sequence identities with a cut off $\geq 97 \%$ over 450 nucleotides in length were considered significant. The best BLAST hits were those showing the highest percentage similarity for a query coverage of more than $90 \%$. Sequences were aligned with the ClustalX (version 1.83) computer program. ${ }^{10} \mathrm{~A}$ distance tree was obtained using MEGA v.5.1 software with the neighbour-joining algorithm and the Kimura twoparameter model with a gamma distribution. Rhizopus arrhizus (CNRMA8.507) and Rhizopus microsporus (UMIP1124.75) were chosen as out-groups, and the robustness of the branches was evaluated by 1000 bootstrap replications.

\section{Results}

During the study period, we received 251 respiratory samples (median of two samples/patient; range 1-19) from 104 patients (mean age: $56 \pm 14$ years; sex ratio M/F: 1.3). Among these 251 respiratory samples, 129 were sputa and 122 were broncho-tracheal aspirates. Fungal species were found more frequently in cultures of sputa $[76.7 \%(99 / 129)]$ than in cultures of broncho-tracheal aspirates [65.6\% $\quad(80 / 122)]$ $(P=0.053)$. Among the 179 positive samples, 82 (45.8\%) contained mixtures of different fungal species with 33.5\% (60/179) containing two species and $12.3 \%(22 / 179)$ containing $>2$ fungal species. The mixtures were composed of yeast and moulds $(26.3 \%$; 47/179), and different moulds (10.1\%; 18/179).

From the 179 positive samples, we classified 287 fungal colonies (142 moulds and 145 yeasts) based on routine examination (Table 1). We identified 17 yeast genera/species, with Candida albicans and Candida glabrata being the two main species. Based on microscopic features, we identified 11 different genera/species of moulds, with A. fumigatus, Penicillium spp., and NSM being the main species (Table 1 ).

We isolated 24 NSMs in total from 19 patients (Table 2), 22 of which were identified by ITS sequencing. For the remaining two isolates, we failed to obtain pure colonies and therefore to obtain an analyzable chromatogram (Patients 18 and 19, Table 2). Eighteen isolates belonged to the Basidiomycota and six to the Ascomycota (Fig. 1). All sequences were assigned to the species level except for two basidiomycetes (NSMs 2 and 10), which were only identified to the

Table 1 Fungal identification obtained for the 287 isolates upon routine identification.

\begin{tabular}{|c|c|c|c|c|c|c|c|}
\hline Mould & $\begin{array}{l}\text { Aspiration } \\
n=60\end{array}$ & $\begin{array}{l}\text { Sputum } \\
n=83\end{array}$ & $\begin{array}{l}n(\%) \\
n=142\end{array}$ & Yeast & $\begin{array}{l}\text { Aspiration } \\
n=65\end{array}$ & $\begin{array}{l}\text { Sputum } \\
n=80\end{array}$ & $\begin{array}{l}n(\%) \\
n=145\end{array}$ \\
\hline Aspergillus fumigatus & 28 & 22 & $50(35.2)$ & Candida albicans & 31 & 33 & $64(44.1)$ \\
\hline Penicillium sp. & 9 & 22 & $31(21.8)$ & C. glabrata & 16 & 13 & $29(20.0)$ \\
\hline NSM & 15 & 9 & $24(16.9)$ & Geotrichum sp. & 2 & 13 & $15(10.3)$ \\
\hline A. niger & 5 & 8 & $13(9.2)$ & C. kefyr & 4 & 7 & $11(7.6)$ \\
\hline Fusarium sp. & 0 & 5 & $5(3.5)$ & C. Iusitaniae & 2 & 3 & $5(3.4)$ \\
\hline Trichoderma sp. & 1 & 4 & $5(3.5)$ & C. krusei & 2 & 1 & $3(2.1)$ \\
\hline A. flavus & 0 & 4 & $4(2.8)$ & Candida sp. & 2 & 1 & $3(2.1)$ \\
\hline A. versicolor & 0 & 3 & $3(2.1)$ & C. tropicalis & 1 & 1 & $2(1.4)$ \\
\hline Aspergillus sp. & 1 & 1 & $2(1.4)$ & C. inconspicua & 2 & 0 & $2(1.4)$ \\
\hline Alternaria sp. & 0 & 2 & $2(1.4)$ & C. famata & 1 & 1 & $2(1.4)$ \\
\hline Cladosporium sp. & 1 & 1 & $2(1.4)$ & $\begin{array}{l}\text { Saccharomyces } \\
\text { cerevisiae }\end{array}$ & 0 & 2 & $2(1.4)$ \\
\hline Mucorales & 0 & 1 & $1(0.7)$ & Others $^{1}$ & 2 & 5 & $7(4.8)$ \\
\hline
\end{tabular}

${ }^{1}$ C. cantenulata, C. colliculosa, C. guilliermondii, C. norvegensis, C. peliculosa, C. pulcherrima, and Candida sp. one isolate each. Yeast name as provided by API 32C, Biomérieux. 


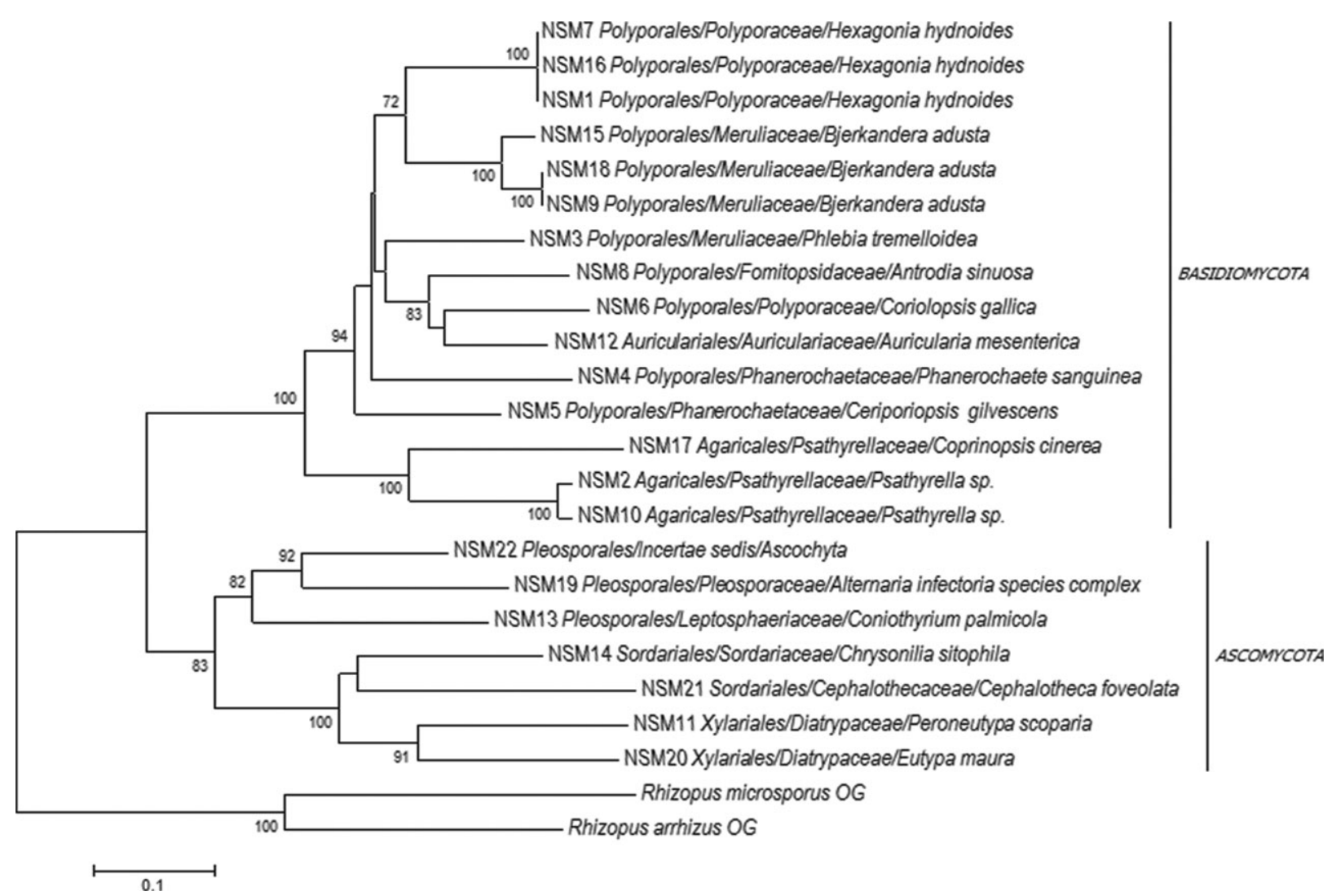

Figure 1 Neighbour-joining tree generated from the internal transcribed spacer (ITS) sequences of the 22 identified non-sporulating moulds listed in Table 2. Bootstrap values are indicated above the branches. Only values above $70 \%$ are shown. The bar indicates the number of substitutions per site.

genus level (Psytharella spp.). The identity of one ascomycete isolate remained uncertain (NSM 22) because its ITS sequence did not correspond to any known taxa. Different NSM species were observed in one sample from Patient 14 (NSMs 16 and 17) and in serial samples from three other patients (Patients 2: NSMs 2 and 3; Patient 3: NSMs 4 and 5; and Patient 17: NSMs 20, 21 and 22). Globally, NSMs were assigned to 16 different genera/species by sequencing, including three that were detected more than once: Hexagonia hydnoides $(n=3)$, Bjerkandera adusta $(n=3)$ and Psathyrella sp $(n=2)$. Among the Ascomycota, no NSM corresponded to non-sporulating A. fumigatus or any other species usually involved in IMD.

Several NSMs were frequently isolated with other moulds or yeast in the same sample (10/24 for other moulds and 6/24 for yeast; Table 2). Of note, NSMs were isolated in five patients with probable IA either concomitantly with A. fumigatus (Patients 15 and 17), or in a subsequent sample (Patients 3, 9 and 17). None of the NSMs were ascribed any specific pathogenic role by clinicians. For the only Mucorales isolate identified (Table 1), the corresponding patient was not diagnosed with IMD and the isolate was not further investigated. This also applied to the five Fusarium sp isolates, one of which was obtained from a patient without probable IMD and the other four were isolated along with A. fumigatus in patients with probable IA.

Given that no NSM was recovered twice in patients with $\geq 2$ samples, we examined whether this was also true for A. fumigatus. Out of 25 patients (16 probable IAs; nine without IA) with $\geq 2$ samples available and at least one A. fumigatus isolated, 10 had $\geq 2$ A. fumigatus positive samples and six only one (Fisher's exact test, $P$ : $0.097, \mathrm{~ns})$, confirming that A. fumigatus more than twice was not taken into account by the clinicians when classifying the patients according to the EORTC/MSG definitions.

\section{Discussion}

Little attention has been paid to the details of the mycological results collected for the diagnosis of IMD. Indeed, neither the number of mould-positive cultures 


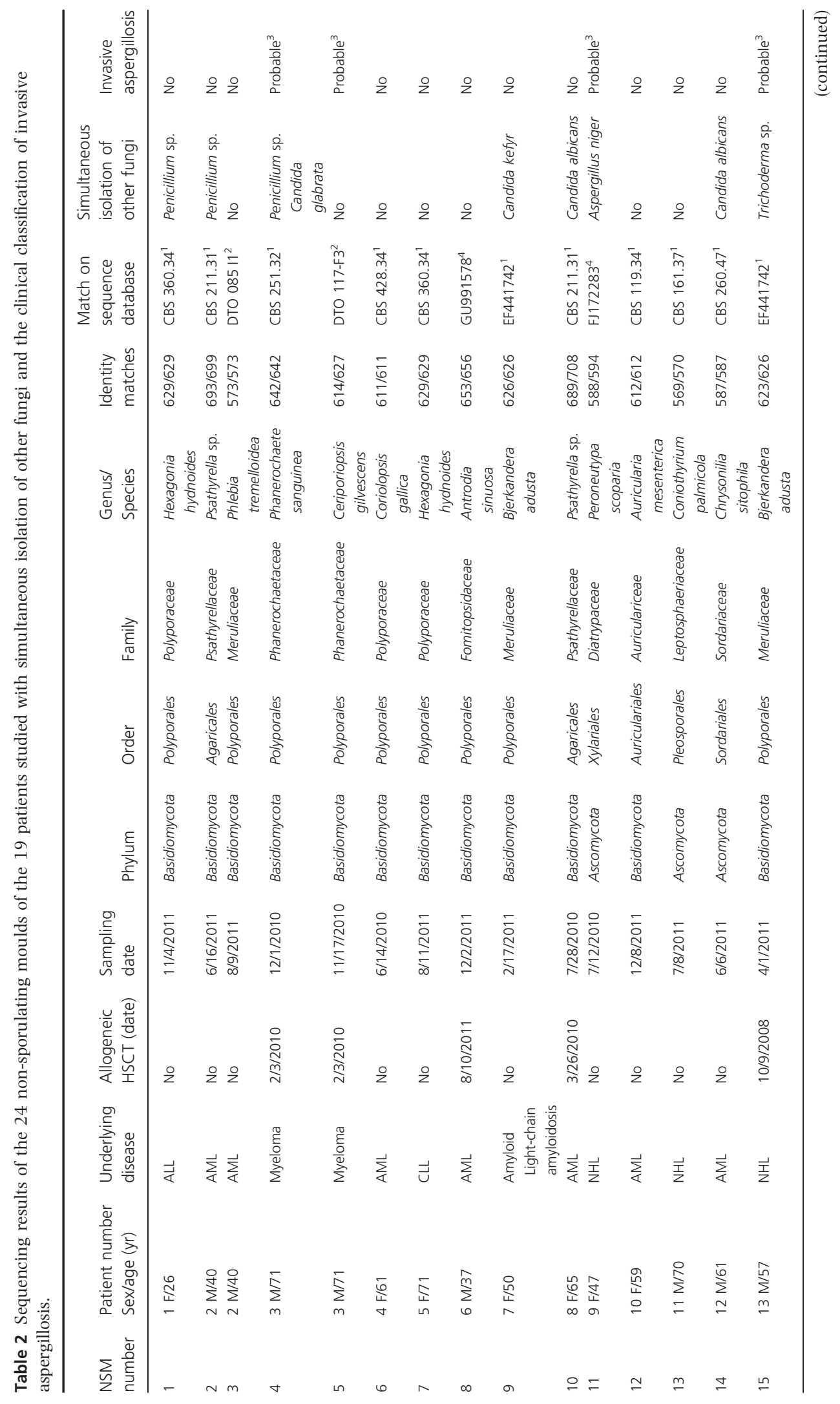




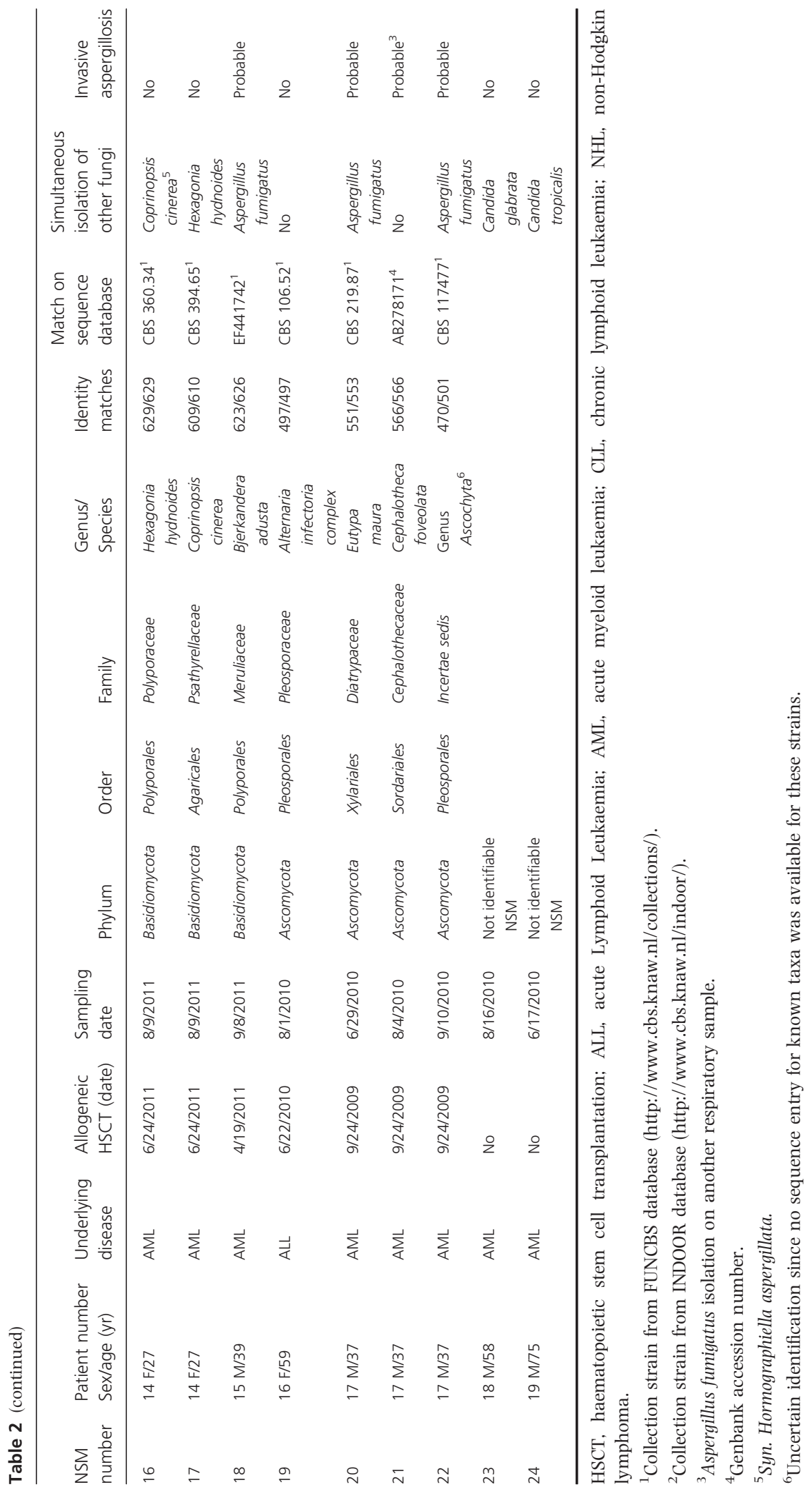


nor the presence of a single mould species recovered in culture are considered in the EORTC/MSG definitions of $\mathrm{IMD}^{1}$ and are therefore not reported. ${ }^{2,4}$ By focusing specifically on NSMs, we clearly show that the fungal species isolated from respiratory specimens of immunocompromised patients are highly variable, mixtures of culturable species are frequent and reproducibility over serial samples is often lacking. The frequency with which different moulds were isolated from the same sample $(10.1 \% ; 18 / 179)$ in the present study was probably greatly underestimated, because: (i) some species can interfere with the growth of other species and pure cultures are often difficult to obtain; (ii) we did not identify some isolates at the species levels (e.g. Penicillium spp.); (iii) some of the isolates identified actually belong to complexes of species (e.g. A. niger); and (iv) many species cannot grow on Sabouraud medium. In addition to being frequently mixed, moulds were also very diverse in terms of the species involved. Ten different genera/species of mould were identified morphologically by microscopy and 22 sequenced NSMs were assigned to 16 different genera/ species. An additional advantage of sequencing NSMs is that it can rule out the presence of nonsporulating pathogenic species, such as a non-sporulating A. fumigatus. ${ }^{7}$

The epidemiology of IMD in haematology patients has changed in recent years with new populations at risk, such as patients with lymphoproliferative diseases, ${ }^{2}$ long-term steroid therapy as the main risk factor $^{11,12}$ and breakthough infections under antifungal prophylaxis. ${ }^{13}$ Galactomannan detection tends to be less sensitive in these patients. As a consequence, direct examination and culture are more often the only mycological criteria to assess probable IA. ${ }^{1}$ Therefore, the interpretation of cultures of mixed moulds or those containing moulds with no known pathogenic role should be revised in immunocompromised patients investigated for fever or febrile neutropenia. NSMs are often deemed clinically irrelevant because the simplest and most widely accepted explanation for their presence is environmental contamination. However, some NSMs from the basidiomycetes ${ }^{14}$ as well as hyalo- and phaeohyphomycosis ${ }^{15}$ are now thought to be potential pathogens.

A common although unproven criterion to ascribe a pathogenic role to a new species, is the recovery of the same species in serial samples, ${ }^{8}$ with the hypothesis that persistent species are more likely to be pathogenic. Whether a NSM was isolated more than once is rarely specified in case reports for unusual or newly reported species. ${ }^{14}$ In our study, we did not grow
NSMs of the same species in samples from the same patient more than once, which argues against the perennial presence of the species in the respiratory tract. However, persistent isolation of A. fumigatus from patients with a final diagnosis of probable IA was not observed in our population either. Moreover, it remains impossible to determine whether a cultivable A. fumigatus was recently acquired or is a long-term resident of the bronchi with higher pathogenic potential. Even with well-established genotyping methods and extensive sampling, the timing of acquisition of A. fumigatus cannot be elucidated ${ }^{16,17}$ and that would be probably the same for NSMs.

An argument for considering NSMs in immunocompromised patients is the results of next generation sequencing (NGS). This technology shows that the association of several moulds is the rule in respiratory specimens and not the exception, with the coining of the term 'mycobiome'. ${ }^{18,19}$ The mycobiome should be explored in immunocompromised patients with haematological disorders, as reported for asthma, ${ }^{20}$ cystic fibrosis patients ${ }^{21}$ and lung transplant recipients. $^{22}$ Currently, the main advantage of cultures over NGS, besides the lower cost and workload, is that cultures select viable moulds and not only DNA, which, on the other hand, can introduce bias in the analysis of species diversity.

The isolation of non-aspergillus moulds in respiratory specimens from haematology patients should be accorded the same significance as yeast is in some centres. Indeed, although some authors did not find any correlation between Candida and death from pneumonia, ${ }^{23}$ others found that Candida either limits, ${ }^{24}$ or promotes $^{25}$ bacterial lung injury. The isolation of several moulds could thus be considered an indirect sign of an altered respiratory epithelium, as imbalance in the intestinal mycobiome is thought to play a role in gastro-intestinal graft-vs.-host disease. ${ }^{26}$ Alterations of the respiratory epithelium may also increase the risk of A. fumigatus conidia being trapped in the bronchi. Additionally, some non-fumigatus moulds may be involved in asthma or chronic cough, which can also indicate altered or sensitised bronchi. ${ }^{14}$

In conclusion, the fungi present in respiratory specimens of immunocompromised patients are very diverse and can be regarded as part of a mycobiome. Changes to this mycobiome over time should be considered more clinically relevant than the isolation of a particular species at a given time. In future epidemiological studies on IA (at least until more studies investigating the pulmonary mycobiome in patients with respiratory diseases are performed), the number of samples 
yielding A. fumigatus colonies and the presence of other moulds should be specified. This may help us to understand, at least in part, the involvement of nonaspergillus species in these patients. Protocols should be established to define the number of respiratory samples and all identified fungi should be declared. This will also help to understand the significance of the galactomannan detection in respiratory specimens since several fungal species can produce this antigen.

\section{Acknowledgments}

This study was carried out as part of our routine work. We thank all the staff of the Henri Mondor Mycology Laboratory for their technical support, all the clinicians from the hospital for their cooperation in collecting respiratory samples and Pr Françoise Dromer for her critical reading of the manuscript.

\section{Conflicts of interest}

We have no conflict of interest regarding the software, products, or concepts used in this study.

\section{References}

1 De Pauw B, Walsh TJ, Donnelly JP et al. Revised definitions of invasive fungal disease from the European Organization for Research and Treatment of Cancer/Invasive Fungal Infections Cooperative Group and the National Institute of Allergy and Infectious Diseases Mycoses Study Group (EORTC/MSG) Consensus Group. Clin Infect Dis 2008; 46: $1813-21$.

2 Lortholary O, Gangneux JP, Sitbon K et al. Epidemiological trends in invasive aspergillosis in France: the SAIF network (2005-2007). Clin Microbiol Infect 2011; 17: 1882-9.

3 Slavin M, van Hal S, Sorrell TC et al. Invasive infections due to filamentous fungi other than Aspergillus: epidemiology and determinants of mortality. Clin Microbiol Infect 2015; 21: 490.e1-490.e10. Epub 2015 Jan 14.

4 Steinbach WJ, Marr KA, Anaissie EJ et al. Clinical epidemiology of 960 patients with invasive aspergillosis from the PATH Alliance registry. J Infect 2012; 65: 453-64.

5 Schoch CL, Seifert KA, Huhndorf S et al. Nuclear ribosomal internal transcribed spacer (ITS) region as a universal DNA barcode marker for Fungi. Proc Natl Acad Sci USA 2012; 109: 6241-6.

6 Fisher MC, Henk DA, Briggs CJ et al. Emerging fungal threats to animal, plant and ecosystem health. Nature 2012; 484: 186-94.

7 Callister ME, Griffiths MJ, Nicholson AG et al. A fatal case of disseminated aspergillosis caused by a non-sporulating strain of Aspergillus fumigatus. J Clin Pathol 2004; 57: 991-2.

8 Arendrup MC, Bille J, Dannaoui E, Ruhnke M, Heussel CP, Kibbler C. ECIL-3 classical diagnostic procedures for the diagnosis of invasive fungal diseases in patients with leukaemia. Bone Marrow Transplant 2012; 47: 1030-45.
9 White TJ, Bruns T, Lee S, Taylor JW. Amplification and direct sequencing of fungal ribosomal RNA genes for phylogenetics. In: Innis MA, Gelfand DH, Sninsky JJ, White TJ (eds), PCR Protocols: A Guide to Methods and Applications. New York: Academic Press Inc, 1990: 315-22.

10 Thompson JD, Higgins DG, Gibson TJ. CLUSTAL W: improving the sensitivity of progressive multiple sequence alignment through sequence weighting, position-specific gap penalties and weight matrix choice. Nucleic Acids Res 1994; 22: 4673-80.

11 Cordonnier C, Botterel F, Ben Amor R et al. Correlation between galactomannan antigen levels in serum and neutrophil counts in haematological patients with invasive aspergillosis. Clin Microbiol Infect 2009; 15: 81-6.

12 Marr KA, Carter RA, Boeckh M, Martin P, Corey L. Invasive aspergillosis in allogeneic stem cell transplant recipients: changes in epidemiology and risk factors. Blood 2002; 100: 4358-66.

13 Lerolle N, Raffoux E, Socie G et al. Breakthrough invasive fungal disease in patients receiving posaconazole primary prophylaxis: a 4-year study. Clin Microbiol Infect 2014; 20: 0952-9; doi:10.1111/14690691.12688

14 Chowdhary A, Kathuria S, Agarwal K, Meis JF. Recognizing filamentous basidiomycetes as agents of human disease: A review. Med Mycol 2014; 52: 782-97.

15 Naggie S, Perfect JR. Molds: hyalohyphomycosis, phaeohyphomycosis, and zygomycosis. Clin Chest Med 2009; 30: 337-53, vii-viii.

16 Alanio A, Cabaret O, Sitterle E et al. Azole preexposure affects the Aspergillus fumigatus population in patients. Antimicrob Agents Chemother 2012; 56: 4948-50.

17 Bart-Delabesse E, Cordonnier C, Bretagne S. Usefulness of genotyping with microsatellite markers to investigate hospital-acquired invasive aspergillosis. J Hosp Infect 1999; 42: 321-7.

18 Cui L, Morris A, Ghedin E. The human mycobiome in health and disease. Genome Med 2013; 5: 63.

19 Ghannoum MA, Jurevic RJ, Mukherjee PK et al. Characterization of the oral fungal microbiome (mycobiome) in healthy individuals. PLoS Pathog 2010; 6: e1000713.

20 van Woerden HC, Gregory C, Brown R, Marchesi JR, Hoogendoorn B, Matthews IP. Differences in fungi present in induced sputum samples from asthma patients and non-atopic controls: a community based case control study. BMC Infect Dis 2015; 13: 69.

21 Delhaes L, Monchy S, Frealle E et al. The airway microbiota in cystic fibrosis: a complex fungal and bacterial community-implications for therapeutic management. PLOS ONE 2012; 7: e36313.

22 Charlson ES, Diamond JM, Bittinger K et al. Lung-enriched organisms and aberrant bacterial and fungal respiratory microbiota after lung transplant. Am J Respir Crit Care Med 2012; 186: 536-45.

23 Meersseman W, Lagrou K, Spriet I et al. Significance of the isolation of Candida species from airway samples in critically ill patients: a prospective, autopsy study. Intensive Care Med 2009; 35: $1526-31$.

24 Mear JB, Gosset P, Kipnis E et al. Candida albicans airway exposure primes the lung innate immune response against Pseudomonas aeruginosa infection through innate lymphoid cell recruitment and interleukin-22-associated mucosal response. Infect Immun 2014; 82: 306-15.

25 Hamet M, Pavon A, Dalle F et al. Candida spp. airway colonization could promote antibiotic-resistant bacteria selection in patients with suspected ventilator-associated pneumonia. Intensive Care Med 2012; 38: $1272-9$.

26 van der Velden WJ, Netea MG, de Haan AF, Huls GA, Donnelly JP, Blijlevens NM. Role of the mycobiome in human acute graft-versushost disease. Biol Blood Marrow Transplant 2013; 19: 329-32. 